\title{
A class of linear operators
}

\section{Patrick J. Browne}

We begin by considering an $n \times n$ positive matrix measure $\mu_{i j}$ together with its associated Hilbert function space $L^{2}\left(\mu_{i j}\right)$ - this space consisting of all $n$-tuples of Borel functions $\left(F_{1}, \ldots, F_{n}\right)$ such that $\int_{-\infty}^{\infty} \sum_{i, j=1}^{n} F_{i}(t) \overline{F_{j}(t)} d \mu_{i j}(t)<\infty$. The multiplication operator, $T$, in this space has domain

$$
\left\{\left(F_{1}, \ldots, E_{n}\right) \in L^{2}\left(\mu_{i j}\right) \mid\left(t F_{1}(t), \ldots, t F_{n}(t)\right) \in L^{2}\left(\mu_{i j}\right)\right\} .
$$

For a point $\left(F_{1}, \ldots, F_{n}\right)$ in this domain

$$
T\left(F_{1}(t), \ldots, F_{n}(t)\right)=\left(t F_{1}(t), \ldots, t F_{n}(t)\right) \text {. }
$$

We then take an arbitrary self adjoint operator $T$ on a Hilbert space $H$ and produce a collection of $n \times n$ positive matrix measures $\left(\mu_{i j}^{N}\right)$ and a unitary map $U: H \rightarrow \sum_{N} L^{2}\left(\mu_{i j}^{N}\right)$ such that $\left(U T U^{-1}\right)$, relative to the space $L^{2}\left(\mu_{i j}^{N}\right)$, is the multiplication operator in that space. This generalizes the theory of spectral representation given by Dunford and Schwartz. We show that when $H=L^{2}(S, \Sigma, v)$ where $(S, \Sigma, \nu)$ is a positive measure space, and when $T$ satisfies a certain condition, the unitary map $U$ can be written as an integral operator. This part of the thesis has been published separately [1].

Self adjoint linear difference operators (corresponding to Sturm-Liouville operators) are discussed in detail.

Received 29 January 1971. Thesis presented to the Flinders University of South Australia, June 1970. Degree approved, December 1970. Supervisor: Professor D.B. Sears. 
Finally we use the representation theory discussed above to produce spectral theorems for these difference operators and to give new proofs of the spectral theorems for self adjoint differential operators with one or two singularities.

\section{Reference}

[1] Patrick J. Browne, "Self adjoint operators and matrix measures", Bulz. Austral. Math. Soc. 4 (1971), 289-305. 